Monográfico: ENDOUROLOGÍA Y LÁSER

Arch. Esp. Urol., 61, 9 (1.130-1.134), 2008

\title{
COMPLICACIONES DE LA UTILIZACIÓN DEL LÁSER
}

Juan Antonio López García, Iciar Crespo Crespo, Lore Aguirreazaldegui García e lgor Oyarzabal Pérez.

Servicio de Urología. Complejo Hospitalario Donostia. Universidad del Pais Vasco. Guipuzcoa. España.

Resumen.- En este trabajo analizamos las complicaciones de las diferentes aplicaciones del láser en urología. La búsqueda de una menor agresión en un intento de disminuir las complicaciones ha desarrollado esta energía en las patologías mas frecuentes de Urología. La utilización en la fragmentación de cálculos ureterales con láser Holmium ha modificado los algoritmos terapéuticos. En patología prostática analizamos las diferentes opciones desde la enucleación (Holep) y la fotovaporización con láser verde revisando las referencias bibliográficas y comparándolos con nuestra experiencia. Existen otras aplicaciones terapéuticas que utilizan esta energía como instrumento de corte en estenosis uretral, ureteral, unión pieloureteral o cervicotomía.
Palabras clave: Complicaciones del láser en urología. Láser.

Summary.- In this paper we analyze the complications of various applications of laser in urology. The search of a minor aggression trying to diminish complications have lead the development of the use of this energy in the most frequent urologic pathologies. Its use in the fragmentation of ureteral stones has modified the therapeutic algorithms. In prostatic diseases we analyze the various options from enucleation (HoLEP) to green laser photovaporization, reviewing the bibliographic references and comparing them with our experience. There are other therapeutic applications that use this energy as a cutting instruments for urethral, ureteral, ureteropyelic junction stenosis, or bladder neck section.

Keywords: Complications laser surgery. Laser.

\section{INTRODUCCIÓN}

El conocimiento acerca de los diferentes tipos de lasers y su potencial uso, ha hecho que en los últimos años se incrementen las indicaciones.

Sería razonable pensar que la aplicación de laser como instrumento de corte, "bisturi", proporciona unas ventajas evidentes; no contacto, menor sangrado, mayor precisión con mejor visualización, menor edema y dolor postoperatorio, así como menor número de complicaciones. Sin embargo estas ventajas ampliamente señaladas, hacen que se desborden las expectativas y se incremente un entusiasmo que puede enturbiar o enmascarar la realidad clínica en los estudios a largo plazo.

Las aplicaciones de la energía láser en Urología tienen su máxima utilización, en el momento actual, en la litiasis (ureteral e intrarrenal RIRS), patología obstructiva prostática con la resección y enucleación (Holep) con láser holmium, y vaporización con KTP (potassium -titanyl phos- 
phate, green light), estenosis de uretra, endopielotomía y resección de tumores del tracto urinario superior, con láser Holmium y en lesiones cutáneas peneanas con láser $\mathrm{CO}_{2}$ y $\mathrm{Nd}$ :YAG.

En este trabajo vamos a intentar analizar las complicaciones, contrastando nuestra práctica diaria con los diferentes láseres, alexandrite y holmium en la litiasis y en patología prostática, vaporización con HPS (nueva generación de KTP) y otras patologías estenosis uretra y de unión pieloureteral, comparándolos con las referencias bibliográficas actuales.

\section{LITIASIS}

La aplicación del láser holmiun (Ytrium-AuminiunGarnet laser) en la fragmentación de cálculos ha sido ampliamente señalada en numerosos estudios, demostrando su eficacia $(1,2,3,4)$. Indudablemente se han mejorado los índices de fragmentación de láser precedentes, alexandrite, el de colorante pulsátil (Candela) y su uso hoy está estandarizado en la aplicación de fragmentación litiásica en uréter mediante URS (rígida o flexible) y en litiasis renal residual mediante cirugía endoscópica intrarrenal con instrumentos flexibles RIRS $(3,4,5,6,7)$.

La aplicación de láser doble pulso (FREDDY laser) y el Erbium:YAG láser no han demostrado superar al holmium considerado como "gold standard" de la litotricia intraureteral (5), no obstante su bajo coste le posiciona como una opción razonable, aunque ha demostrado menor eficacia de fragmentación en litiasis de gran dureza.

Refiriéndonos a las complicaciones de la aplicación con láser holmium en la litiasis ureteral, estarán condicionadas en gran manera por parámetros como grado de hidronefrosis, tamaño del cálculo, localización, grado de impactación (7). Indudablemente estos factores condicionan el propio acto endoscópico y al prolongarse la fragmentación con láser, en casos de cálculos grandes o impactados, puede condicionar un aumento de sangrado o lesión ureteral por pérdida de visión. Estas serían las principales complicaciones directamente atribuibles a la fuente de energía. Al ser una energía de contacto, es necesaria una correcta visión para lograr que la fibra pueda fragmentar el cálculo, en aquellos casos de pérdida de visión las posibilidades de perforación ureteral aumentan.

Otra de las complicaciones señaladas sería el ascenso o retropulsión del cálculo a las cavidades renales tras su desimpactación, este hecho aún siendo menor que con las energías mecánicas, es una complicación relativamente frecuente que nos obliga a la utilización de instrumentos flexibles para acceder a los diferentes cálices o a la utilización de litotricia extracorpórea por ondas de choque $(8,9,10,11)$.

El índice de complicaciones es mayor en el uréter proximal $8,2 \%$ frente al distal $4,2 \%$ y el grado de impactación $10,5 \%$ frente a la no impactación $3,2 \%$ no encontrando diferencias según edad, sexo, grado de hidronefrosis, o tamaño del cáculo $(7,8)$. La proximidad excesiva, por debajo de 0,5 cm, de la fibra al cálculo puede ocasionar lesión ureteral al no permitir que el agua intermedia absorba parte de la energía $(7,12,13)$.

Como complicaciones intraoperatorias más frecuentes son la hematuria, perforación ureteral y extravasación urinaria, las complicaciones tardías se relacionan más con la propia técnica endoscópica que con el tipo de energía aplicada, la estenosis y fibrosis podrían explicarse por el grado de impactación del cáculo o a las maniobras de fragmentación. Sería difícil no incluir factores isquémicos con la consiguiente fibrosis secundaria debido a la presión del cálculo sobre la pared ureteral y las reacciones inflamatorias que pueden inducir o estimular a una fibrosis secundaria $(7,13)$.

Las cifras de libres de cálculos se correlacionan con el grado de impactación $94,6 \%$ frente $82 \%(2,3)$. Igualmente un menor tiempo de enclavamiento tras la SWL o de permanencia del cálculo en el uréter, disminuirá la fibrosis, edema posterior evitando las complicaciones futuras $(7,8,12)$.

Desde la aplicación del láser Holmium, se ha simplificado el manejo de la litiasis ureteral con cifras de éxito mayores y disminución de la retropulsión frente a las energías mecánicas. En términos generales se han mejorado las cifras de "libres de cálculos" y han disminuido el riesgo de complicaciones (5).

El tratamiento de la litiasis residual mediante ureterorrenoscopia flexible está más condicionada por la dificultad técnica de poder acceder a las diferentes cavidades caliciales. Una vez localizado el cáculo conseguir introducir la fibra de láser holmium y dependiendo del grado de deflexión llegar a estabilizar el cáculo para lograr su fragmentación. En esta situación es frecuente la lesión de la mucosa, provocando hematuria que empeora y dificulta la visión y sin ser una complicación sería, si puede condicionar el resultado final de la intervención. El índice de complicaciones se puede considerar bajo y está sujeto a la experiencia del urólogo.

La utilización con el nefroscopio flexible en el tratamiento de la litiasis renal compleja mediante nefrolitotomía percutánea, permite un mejor manejo logrando acceder a las diferentes cavidades caliciales en busca de litiasis inaccesibles al nefroscopio rígido y su fragmentación con el láser Holmium.

Indudablemente la posibilidad de utilizar sondas flexibles de diferente calibre nos permite un excelente manejo intrarrenal y la posibilidad de acceder sin dificultad a dichas cavidades caliciales.

\section{HBP}

La aplicación de la energía láser en el tratamiento de la hipertrofia prostática benigna, tiene diferentes posibilidades según cual sea el láser utilizado. Como hemos señalado inicialmente esta fuente de energía busca minimizar las complicaciones hemorrágicas. Las principales va- 
riedades de láser que más se utilizan en la actualidad son $\mathrm{Nd}$-Yag, el holmium ytrium aluminium garnet (Ho:Yag) y el de doble frecuencia (Nd-Yag), potassium-titanyl-phosphate (KTP) y su variante de última generación HPS.

HOLEP- La enucleación de la próstata con el láser holmium es hoy considerada como la alternativa a la cirugía con unos resultados funcionales altamente satisfactorios y con una mejora evidente reduciendo la estancia hospitalaria confirmándose como una técnica segura y reproducible $(14,15)$.

Las complicaciones señaladas en las diferentes series señalan un bajo índice de reconversión 2,5\%, durante el inicio o aprendizaje $(16,17)$, problemas con la morcelación alrededor del $1 \%$, este acto, desde nuestro punto de vista, es uno de los mayores inconvenientes de esta técnica, que desluce la elegancia de la enucleación y creemos que es susceptible de mejoría. La perforación capsular $9,6 \%$ (18), lesión vesical 3,9\%, lesión del orificio ureteral $2,1 \%$, se precisa trasfusión en $1,4 \%$, bloqueo vesical por coágulos $0,7 \%$, incontinencia urinaria pasajera en el 10,7\% de los pacientes y se mantiene en un $0,7 \%$, precisan de sondaje vesical tras la retirada del catéter 3,9\%, infección urinaria $3,2 \%$, epididimitis $0,7 \%$, estenosis de meato $2,5 \%$ $(18,19,20,21,22)$.

Sin lugar a dudas uno de los mayores inconvenientes de esta técnica es la dificultad de aprendizaje, precisando de una algo lenta y durante este periodo es cuando ocurren la mayoría de las complicaciones serias. Se ha señalado un mínimo de 50 intervenciones para poder controlar la técnica, este hecho técnico dificulta su difusión. En estudio de Shah $(23,24)$ diferenciando en tres grupos consecutivos de 50 pacientes, el éxito se logra en casi la de los enfermos y se adquiere una buena experiencia a partir de los 50 casos.

Si pensamos que la patología de próstata, es posiblemente una de las más frecuentes a las que se enfrenta el urólogo y que en cualquier Servicio recae en la totalidad de sus miembros, resulta difícil soportar un aprendizaje tan lento. Al generalizar su uso se incrementa el número de complicaciones y por tanto se abandone o sea sustituido por técnicas más convencionales, adenomectomía retropúbica, RTU de próstata, RTU energía bipolar. A pesar de esto, en un estudio prospectivo comparando estas dos técnicas Holep vs RTU, la enucleación con láser fue significativamente mejor en tiempo con catéter, estancia hospitalaria, pérdida hemática y peor en tiempo operatorio (mayor duración). Los efectos sobre la continencia y potencia sexual fueron similares en ambos grupos, pero tiene menos efectos adversos el grupo de pacientes intervenidos con láser holmium. (Kunntz RM) $(14,17,26)$.

Un factor limitante es el aprendizaje de la técnica y otra de las restricciones señaladas es el precio aunque dado el carácter multifuncional, debido a su posible utilización en diferentes patologías, le hace ser un instrumento de gran utilidad en un servicio de urología.

Otra de las ventajas señaladas al Holep es que se indica independientemente del volumen prostático, siendo sus resultados no sujetos a las variaciones de tamaño prostático.

\section{FOTOVAPORIZACION KTP (Kalium-Titanyl-phosphate)}

La aplicación de láser de Nd:YAG de doble frecuencia, al pasar un haz de $1064 \mathrm{~nm}$ de longitud de onda, a través de un cristal KTP permite transformar este a la mitad $532 \mathrm{~nm}$ actuando en el espectro de la luz verde, lo que le ha dado este nombre "láser verde". Esta longitud de onda es absorbida bien por la hemoglobina y provoca un grado intermedio de vaporización-coagulacion. Los inconvenientes iniciales debido a la lentitud del tejido vaporizado con $80 \mathrm{~W},(27,28)$ se ha mejorado con la aplicación de mayor potencia con el HPS de $120 \mathrm{~W}$, este proporciona una rápida vaporización fototérmica del agua intracelular proporcionando una vaporización fotoselectiva de la próstata (29). La mayoría de los estudios señalan las ventajas de esta técnica, $(28,30,31)$ estancias más cortas, menor sangrado, unido a una difusión mediática bien elaborada ha permitido ser considerada una alternativa emergente con una elevada demanda por los enfermos prostáticos $(32,33)$.

Se han señalado como complicaciones, lesión vesical, lesión de orificios ureterales especialmente con la vaporización del lóbulo medio, perforación capsular y apertura de senos venosos. Como desventajas de la técnica se señala el síndrome irritativo, que en ocasiones se prolonga hasta varios meses y que precisa de medicación antiinflamatoria. La hematuria suele ser frecuente y persiste en el postoperatorio, debiendo ser advertido el paciente que generalmente no espera estas complicaciones. Igualmente se ha señalado la imposibilidad de recogida de tejido para análisis histológico como una debilidad de la técnica no lográndose detectar ese $5 \%$ de carcinoma incidental que aparecen con otros procedimientos pudiéndose beneficiar de un diagnóstico y tratamiento precoz.

En nuestra experiencia las indicaciones deben ser muy ajustadas, siendo eficaz en próstatas de mediano tamaño donde si puede competir con la RTU, no obstante en próstatas mayores se incrementan las complicaciones teniendo peor control de las mismas que con la cirugía endoscópica tradicional. Indudablemente las expectativas, tanto de algunos estudios como a nivel de los propios enfermos, que generan una esperanza de mejora que contrasta con la realidad clínica.

Los estudios comparativos de KTP vs RTU dan mayor número de complicaciones para la resección, obstrucción por coágulos, trasfusión sanguínea, estenosis de meato o esclerosis de cuello vesical. El síntoma irritativo miccional es señalado como el más frecuente manteniéndose en el tiempo hasta seis semanas. En nuestra experiencia hay enfermos que mantienen dicha sintomatología hasta tres meses, precisando durante ese tiempo medicación para aliviar dicha clínica. Como hemos señalado previamente el índice de complicaciones se correlaciona con el volumen, siendo directamente proporcional, motivo por el que en próstatas voluminosas o con lóbulo medio prominente no consideremos esta técnica. Indudablemente las expec- 
tativas generadas por esta técnica, tanto en los urólogos como en los pacientes, contrastan con la realidad clínica. Creemos que es preciso realizar estudios bien diseñados y con datos objetivos a largo plazo, para poder confirmar los resultados iniciales, el tiempo nos dirá el lugar exacto que le corresponde a la fotovaporización.

\section{OTRAS APLICACIONES}

\section{TUMORES UROTELIALES}

El manejo de TUS (TCC) por vía endoscópica, cirugía percutánea o URS estaría indicado en situaciones especiales (monorreno, insuficiencia renal, bilateralidad o comorbilidad médica severa) (34,35). La ablación o electrofulguración con láser es una alternativa, en tumores focales de bajo o grado intermedio, en pacientes seleccionados y que nos obliga a controles endoscópicos periódicos $(36,37)$.

Las complicaciones atribuibles a la utilización con láser serían el sangrado y la perforación, añadiéndose a las complicaciones de la propia técnica endoscópica.

En tumores vesicales no infiltrantes de bajo grado, en aquellos casos de siembras, de pequeño tamaño, poco exofíticas puede aplicarse el láser Nd:YAG. La dificultad señalada es la de no conseguir estudio histológco siendo su principal indicación en tumores recurrentes de bajo grado. Se han descrito como complicaciones la posibilidad de lesión de estructuras vecinas, con perforación de asas intestinales.

\section{ESTENOSIS}

La utilización de láser holmium en estenosis, como instrumento de sección sustituyendo al corte frio puede ser aplicada en estenosis de uretra sobre un tutor o guía, ha sido ampliamente utilizada así como en estenosis ureterales y en endopielotomías $(39,40)$. Las complicaciones que hemos encontrado como más frecuente es la dificultad para dirigir el haz y controlar la profundidad del corte en uréter y uretra. En la sección de la unión pieloureteral encontramos mayor dificultad para realizar el corte que con el asa de Collins así como el láser provoca lesiones del catéter ureteral que nos obliga al cambio del mismo. Este hecho nos condiciona, ya que las maniobras de recolocación del catéter de doble J una vez realizada la sección de la unión pieloureteral aumentan el riesgo de desinserción ureteral.

\section{LESIONES CUTÁNEAS Y CÁNCER DE PENE}

El láser de $\mathrm{CO}_{2}$ con una longitud de onda de $10600 \mathrm{~nm}$ tiene una penetración de 0,01 mm con coagulación de tejidos y vasos de $0,5 \mathrm{~mm}$, tiene un efecto de corte y le hace eficaz para lesiones superficiales. Como desventajas se señalan los límites de profundidad del corte y la ausencia de estudios histológicos, su aplicación en lesiones peneanas, premalignas, (enfermedad de Bowen, eritroplasia de Queyrat, papulosis etc.
El láser de Nd:YAG ha sido utilizado por su mayor pentración de 3-6mm y el laser de Argón, con una longitud de onda de $488 \mathrm{~mm}$ y KTP $(532 \mathrm{~mm})$ han sido aplicados, debido a su afinidad por pigmentos tisulares como hemoglobina y melanina en lesiones cutánea muy pigmentadas.

Como mayor debilidad de estas aplicaciones es la dificultad de obtener estudios histológicos.

\section{CONCLUSIONES}

Las complicaciones derivadas de la aplicación de láser en sus diferentes variantes están sujetas, en gran medida, al adecuado uso de la misma, siendo mas atribuidas a una utilización inadecuada, por déficit de visión, por incremento de la potencia o por indicaciones forzadas que al daño generado por dicha energía. En términos generales la aplicación de láser Holmium en la litiasis ha permitido mejorar sin duda, los resultados de la URS.

En la patología prostática las diferentes opciones, enucleción, fotovaporizacion tienen sus indicaciones y sus limitaciones que es preciso conocer para hacer un uso adecuado de esta tecnología, indudablemente no debemos utilizar de forma sistematica

\section{BIBLIOGRAFÍA Y LECTURAS RECOMENDADAS ("lectura de interés $y^{* *}$ lectura fundamental)}

*1. GRASSO, M.; CHALIK, Y.: "Principles and applications of laser lithotripsy: Experience with the Holmium laser lithtrite". J. Clin. Laser Med. Surg., 16: 3, 1998.

2. MUGIYA, S.; ITO, T.; MARUYAMA, S. y cols.: "Endoscopic features of impacted ureteral stones". J. Urol., 171: 89, 2004.

3. MUGIYA, S.; NAGATA, M.; UN-NO ,T. y cols.: "Endoscopic management of impacted ureteral stones using small caliber ureteroscope and laser lithotrptor". J. Urol., 164: 329, 2000.

4. GRASSO, M.; CONLIN, M.; BAGLEY, D.: "Retrograde uretheropyeloscopic, treatment of $2 \mathrm{~cm}$.or greater upper urinary tract and minor Staghorn calculi".J. Urol., 160: 346, 1998.

5. MARGUET, C.G.; SUNG, J.C.; SPRINGHART, W.P. y cols.: "In Vitro comparison of stone retropulsion and fragmentation of the frequency doubled, double pulse Nd: YAG laser and the holmium: yag laser". J. Urol., 173: 1797, 2005.

6. NAQUI, S.A.A.; KHALIQ, M.; ZAFAR, M.N. y cols.: "Treatment of ureteric stones. Comparison of laser and pneumatic lithotripsy”. B.J.U., 74: 694, 1994.

*7. SEITZ, C.; TANOVIC, E.; KIKIC, Z. y cols.: "Impact of stone size, location, composition, impaction, and hidronephrosis on the efficacy of Holmium: YAG-Laser uretherolithotripsy". Eur. Urol., 52: 1751, 2007.

8. JUNG, H.; NORBY, B.; OSTHER, P.J.: "Retrograde intrarenal stone surgery for extracorporeal shock-wave 
lithotripsy-resistant kidney stones". Scand. J. Urol. Nephol., 40: 380, 2006.

9. EL ANANY; F.G.; HAMMOUDA, H.M.; MAGHRABY, H.A. y cols.: "Retrograde ureteropyeloscopic Holmium laser lithotripsy for large renal calculi". BJU., 88: $850,2001$.

10. DOGAN, H.S.; TEKGUL, S.; AKDOGAN, B. y cols.: "Use of Holmium: YAG laser for ureterolithotripsy in children”. BJU. Int., 94: 131, 2004.

11. EL-ASSMY, A.; HAFEZ, A.T.; ERAKY, I. y cols.: "Safety and outcome rigid ureteroscopy for management of ureteral calculi in children". J. Endourol., 20: 252, 2006.

**12. PIERRE, S.; PREMINGER, G.M.: "Holmium laser for stone management". World J. Urol., 25: 235, 2007.

*13. GEAVLETE, P.; GEORGESCU, D.; NITA, G. y cols.: "Complications of 2735 retrograde semirigid ureteroscopy procedures: A single center experience". J. Endourol., 20: 179, 2006.

*14. KUNTZ, R.M.; AHYAI, S.; LEHRICH, K. y cols.: "Transurethral holmium laser enucleation of the prostate versus transurethral electrocautery resection of the prostate. A randomized prostate: A randomized prospective trial in 200 patients". J. Urol., 172: 1012, 2004

*15. PLANZ, B.; KALEM, T.; SPREGER, C. y cols.: “A prospective randomized study of combined visual laser ablation and transurethral resection of the prostate versus transurethral prostatectomy alone". Urol. Int., 71: 26, 2003.

16. ELZAYAT, E.A.; ELHILALI, M.M.: "Holmium laser enucleation of the prostate (HoLEP): Long term results, reoperation rate, and possible impact of the learning curve". Eur. Urol., 52: 1465, 2007.

**17. KUNTZ, R.M.: "Laser treatment of benign prostatic hyperplasia". World J. Urol., 25: 241, 2007.

*18. WILSON, L.C.; GILLING, P.J.; WILLIAMS, A. y cols.: "A randomised trial comparing Holmium laser enucleation versus transurethral resection in the treatment of prostates larger than 40 grams: Results at 2 years". Eur. Urol., 50: 569, 2006

19. KUNTZ, R.M.; LEHRICH, K.; AHYAI, S.A.: "Holmium Laser enucleation of the prostate versus open prostatectomy for prostates greater than 100 grams: 5 year follow-up results of a randomised clinical trial. Eur. Urol., 53: 160, 2008.

*20. AHYAI, S.A.; LEHRICH, K., KUNTZ, R.M.: "Holmium laser enucleation versus transurethral resection of the prostate: 3 year follow-up results of a randomized clinical trial". Eur. Urol., 52: 1456, 2007.

21. TAN, A.H.; GILLING, P.J.; KENNETT, K.M. y cols.: "A randomized trial comparing holmium laser enucleation of the prostate with transurethral resection of the prostate with transurethral resection of the prostate for the treatment of bladder outlet obstruction secondary to benign prostatic hyperplasia in large glands (40 to 200 grams)". J. Urol., 170: 1270, 2003.

**22. SHAH, H.N.; MAHAJAN, A.P.; HEGDE, S.S. y cols.: "Peri-operative complications of holmium laser enucleation of the prostate: Experience in the first 280 patients and a review of literature". BJU., 100: 94, 2007.

23. SHAH, H.N.; MAHAJAN, A.P.; SODHA, H.S. y cols.: "Prospective evaluation of the learning curve for Holmium laser enucleation of the prostate". J. Urol., 177:
1468, 2007.

24. HEMENDRA, N.; SHAH, S.S.; HEGDE, J.N. y cols.: "Simultaneous transurethral cystolithotripsy with Holmium laser enucleation of the prostate: A prospective feasibility study and review of literature". BJU., 99: 595, 2006

25. THORSTEN, B.; THOMAS, R.W.; GANZER, H.R. y cols.: "Revolix vaporesection of the prostate: Initial results of 54 patients with a 1 year follow-up". World J. Urol., 25: 257, 2007.

26. KUNTZ, R.M.; LEHRICH, K.; AHYAI, S.: "Transurethral Holmium laser enucleation of the prostate compared with transvesical open prostatectomy: 18-month followup of a randomized trial”. J. Endourol., 18: 189, 2004.

*27. VERGER-KUHNKE, A.B.; REUTER, M.A.; EPPLE, W. y cols.: "La vaporación fotoselectiva de la HPB con el KTP-LASER (Kalium-titanyl-phosphat) de 80 watt y la RTU-P de baja presión hidráulica: Experiencia en 230 casos". Arch. Esp. Urol., 60: 167, 2007.

*28. MALEK, R.S.; KUNTZMAN, R.S.; BARRET, D.M: "Photoselective potassium-titanyl-phosphate laser vaporzaction of the benign obstructive prostate: Observations on long-term outcomes". J. Urol., 174: 1344, 2005.

29. ALEXIS, E.; TE, M.D.: The next generation in laser treatnents and the role of the green light high-performance system laser". Rev. Urol., 8: 524, 2006.

30. BOUCHIER-HAYES, D.M.; ANDERSON, P.; VAN APPLEDORN, s. y cols.: "KTP Laser versus Transuretharl Resection: Early results of a randomized trial”. J. Endourol., 20: 580, 2006.

31. HEINRICH, E.; SCHIEFELBEIN F.; SCHOEN, G.: "Technique and short-term outcome of green ligh laser (KTP, $80 \mathrm{~W})$. Vaporisation of the Prostate". Eur. Urol., 52: $1632,2007$.

32. COURTNEY, K.P.; LANDMAN, J.: "Lasers in the upper urinary tract for non-stone disease". World J. Urol., 25: 249, 2007.

33. ASSIMOS, D.G.; HALL, M.C.; MARTIN, J.H.: "Ureteroscopic management of patients with upper tract transitional cell carcinoma". Urol. Clin. North Am., 27: $751,2000$.

34. CHEN, G.L.; BAGLEY, D.H.: "Uretherscopic management of upper tract transitional cell carcinoma in patients with normal contralateral kidneys". J. Urol., 164: 1173,2000 .

35. BOORJIAN, S.; NG, C.; MUNVER, R. y cols.: "Impact of delay to nephroureterectomy for patients undergoing ureteroscopic biopsy and laser tumor ablation of upper tract transitional cell carcinoma". Urology, 66: 283, 2005.

36. FUJIMOTO, H.; TOBISU, K.I.; MIZUTANI, t. y cols.: "Trasitional cell carninoma of the upper urinary tract: Analysis of morphology and distrbution for surgical management". Japanese Journal of Clinical Oncology, 23: 303, 1993.

37. PONSKY, L.E.; STREEM, S.B.: "Retrograde endopyelotomy: A comparative study of hotwire balloon and ureteroscopic laser". J. Endourol., 20: 823, 2006.

38. THORSTEN, B.; THOMAS, R.W.; HERRMANN, C.: "Bladder neck incision using a $70 \mathrm{~W} 2$ micron continuous wave laser (RevoLix)". World. J. Urol., 25: 263, 2007. 